\title{
Factors associated with syrinx size in pediatric patients treated for Chiari malformation type I and syringomyelia: a study from the Park-Reeves Syringomyelia Research Consortium
}

\author{
Andrew T. Hale, PhD, ${ }^{1,2}$ P. David Adelson, MD, ${ }^{3}$ Gregory W. Albert, MD, MPH, ${ }^{4}$ \\ Philipp R. Aldana, MD, ${ }^{5}$ Tord D. Alden, MD, ${ }^{6}$ Richard C. E. Anderson, MD, ${ }^{7}$ David F. Bauer, MD, ${ }^{8}$ \\ Christopher M. Bonfield, MD, ${ }^{2,9}$ Douglas L. Brockmeyer, MD, ${ }^{10}$ Joshua J. Chern, MD, PhD, ${ }^{11}$ \\ Daniel E. Couture, MD, ${ }^{12}$ David J. Daniels, MD, PhD, ${ }^{13}$ Susan R. Durham, MD, MS, ${ }^{14}$ \\ Richard G. Ellenbogen, MD, ${ }^{15}$ Ramin Eskandari, MD, ${ }^{16}$ Timothy M. George, MD, ${ }^{17}$ \\ Gerald A. Grant, MD, ${ }^{18}$ Patrick C. Graupman, MD, ${ }^{19}$ Stephanie Greene, MD, ${ }^{20}$ \\ Jeffrey P. Greenfield, MD, PhD, ${ }^{21}$ Naina L. Gross, MD, ${ }^{22}$ Daniel J. Guillaume, MD, MS, ${ }^{23}$ \\ Gregory G. Heuer, MD, PhD, ${ }^{24}$ Mark lantosca, MD, ${ }^{25}$ Bermans J. Iskandar, MD, ${ }^{26}$ \\ Eric M. Jackson, MD, ${ }^{27}$ James M. Johnston, MD, ${ }^{28}$ Robert F. Keating, MD, ${ }^{29}$ \\ Jeffrey R. Leonard, MD, ${ }^{30}$ Cormac O. Maher, MD, ${ }^{31}$ Francesco T. Mangano, DO, ${ }^{32}$ \\ J. Gordon McComb, MD, ${ }^{33}$ Thanda Meehan, RN, ${ }^{34}$ Arnold H. Menezes, MD, ${ }^{35}$ Brent O'Neill, MD, ${ }^{36}$ \\ Greg Olavarria, MD, ${ }^{37}$ Tae Sung Park, MD, ${ }^{34}$ John Ragheb, MD, ${ }^{38}$ Nathan R. Selden, MD, PhD, ${ }^{39}$ \\ Manish N. Shah, MD, ${ }^{40}$ Matthew D. Smyth, MD, ${ }^{34}$ Scellig S. D. Stone, MD, PhD, ${ }^{41}$ \\ Jennifer M. Strahle, MD, ${ }^{34}$ Scott D. Wait, MD, ${ }^{42}$ John C. Wellons, MD, MSPH, ${ }^{2,9}$ \\ William E. Whitehead, MD, MSc, ${ }^{43}$ Chevis N. Shannon, DrPH, MBA, MPH, ${ }^{2,9}$ and \\ David D. Limbrick Jr., MD, PhD, ${ }^{34}$ for the Park-Reeves Syringomyelia Research \\ Consortium Investigators
}

${ }^{1}$ Vanderbilt University School of Medicine, Medical Scientist Training Program, Nashville, Tennessee; ${ }^{2}$ Surgical Outcomes Center for Kids, Monroe Carell Jr. Children's Hospital of Vanderbilt University, Nashville, Tennessee; '3Division of Pediatric Neurosurgery, Barrow Neurological Institute at Phoenix Children's Hospital, Phoenix, Arizona; 'Division of Neurosurgery, Arkansas Children's Hospital, Little Rock, Arkansas; ${ }^{2}$ Division of Pediatric Neurosurgery, University of Florida College of Medicine, Jacksonville, Florida; ${ }^{6}$ Division of Pediatric Neurosurgery, Ann and Robert H. Lurie Children's Hospital of Chicago, Illinois; ' 7 Division of Pediatric Neurosurgery, Department of Neurological Surgery, Children's Hospital of New York, Columbia-Presbyterian, New York, New York; ${ }^{8}$ Department of Neurosurgery, Dartmouth-Hitchcock Medical Center, Lebanon, New Hampshire; ${ }^{9}$ Division of Pediatric Neurosurgery, Monroe Carell Jr. Children's Hospital of Vanderbilt University, Nashville, Tennessee; ${ }^{10}$ Division of Pediatric Neurosurgery, Primary Children's Hospital, Salt Lake City, Utah; ${ }^{11}$ Division of Pediatric Neurosurgery, Children's Healthcare of Atlanta University, Atlanta, Georgia; ${ }^{12}$ Department of Neurological Surgery, Wake Forest University School of Medicine, Winston-Salem, North Carolina; ${ }^{13}$ Department of Neurosurgery, Mayo Clinic, Rochester, Minnesota; ${ }^{14}$ Department of Neurosurgery, University of Vermont, Burlington, Vermont; ${ }^{15}$ Division of Pediatric Neurosurgery, Seattle Children's Hospital, Seattle, Washington; ${ }^{16}$ Department of Neurosurgery, Medical University of South Carolina, Charleston, South Carolina; ${ }^{17}$ Division of Pediatric Neurosurgery, Dell Children's Medical Center, Austin, Texas; ${ }^{18}$ Division of Pediatric Neurosurgery, Lucile Packard Children's Hospital, Palo Alto, California; ${ }^{19 D i v i s i o n ~ o f ~ P e d i a t r i c ~ N e u r o s u r g e r y, ~ G i l l e t t e ~ C h i l d r e n ' s ~ H o s p i t a l, ~ S t . ~ P a u l, ~ M i n n e s o t a ; ~}$ ${ }^{20}$ Division of Pediatric Neurosurgery, Children's Hospital of Pittsburgh, University of Pittsburgh Medical Center, Pittsburgh, Pennsylvania; ${ }^{21}$ Department of Neurological Surgery, Weill Cornell Medical College, NewYork-Presbyterian Hospital, New York, New York; ${ }^{22}$ Department of Neurosurgery, University of Oklahoma Health Sciences Center, Oklahoma City, Oklahoma;

ABBREVIATIONS $\mathrm{AP}=$ anteroposterior; $\mathrm{CM}-\mathrm{I}=$ Chiari malformation type I; $\mathrm{CM}-\mathrm{I}+\mathrm{SM}=\mathrm{CM}-\mathrm{I}$ with $\mathrm{SM} ; \mathrm{CPH}=$ Cox proportional hazards; $\mathrm{CXA}=$ clivus canal angle; $\mathrm{FOHR}$ = frontal-occipital horn ratio; $\mathrm{PB}-\mathrm{C} 2$ distance = distance from the ventral dura perpendicular to the line between the basion and C2; PFD = posterior fossa decompression; PFDD = PFD with duraplasty; PRSRC = Park-Reeves Syringomyelia Research Consortium; SM = syringomyelia.

SUBMITTED August 23, 2019. ACCEPTED January 7, 2020.

INCLUDE WHEN CITING Published online March 6, 2020; DOI: 10.3171/2020.1.PEDS19493. 


\begin{abstract}
${ }^{23}$ Department of Neurosurgery, University of Minnesota Medical School, Minneapolis, Minnesota; ${ }^{24}$ Division of Pediatric Neurosurgery, Children's Hospital of Pennsylvania, Perelman School of Medicine, University of Pennsylvania, Philadelphia, Pennsylvania; ${ }^{25}$ Department of Neurosurgery, Penn State Milton S. Hershey Medical Center, Hershey, Pennsylvania; ${ }^{26}$ Department of Neurological Surgery, University of Wisconsin at Madison, Wisconsin; ${ }^{27}$ Department of Neurosurgery, Johns Hopkins University School of Medicine, Baltimore, Maryland; ${ }^{28}$ Division of Pediatric Neurosurgery, University of Alabama at Birmingham, Alabama; ${ }^{29}$ Department of Neurosurgery, Children's National Medical Center, Washington, DC; ${ }^{30}$ Division of Pediatric Neurosurgery, Nationwide Children's Hospital, Columbus, Ohio; ${ }^{31}$ Department of Neurosurgery, University of Michigan, Ann Arbor, Michigan; ${ }^{32}$ Division of Pediatric Neurosurgery, Cincinnati Children's Medical Center, Cincinnati, Ohio; ${ }^{33}$ Division of Pediatric Neurosurgery, Children's Hospital of Los Angeles, California; ${ }^{34}$ Department of Neurological Surgery, Washington University in St. Louis School of Medicine, St. Louis, Missouri; ${ }^{35}$ Department of Neurosurgery, University of lowa Hospitals and Clinics, lowa City, lowa; ${ }^{36}$ Department of Neurosurgery, Children's Hospital Colorado, Aurora, Colorado; ${ }^{37}$ Division of Pediatric Neurosurgery, Arnold Palmer Hospital for Children, Orlando, Florida; ${ }^{38}$ Department of Neurological Surgery, University of Miami Miller School of Medicine, Miami, Florida; ${ }^{39}$ Department of Neurological Surgery and Doernbecher Children's Hospital, Oregon Health \& Science University, Portland, Oregon; ${ }^{40}$ Division of Pediatric Neurosurgery, McGovern Medical School, Houston, Texas; ${ }^{41}$ Division of Pediatric Neurosurgery, Boston Children's Hospital, Boston, Massachusetts; ${ }^{42}$ Carolina Neurosurgery \& Spine Associates, Charlotte, North Carolina; and ${ }^{43}$ Division of Pediatric Neurosurgery, Texas Children's Hospital, Houston, Texas
\end{abstract}

OBJECTIVE Factors associated with syrinx size in pediatric patients undergoing posterior fossa decompression (PFD) or PFD with duraplasty (PFDD) for Chiari malformation type I (CM-I) with syringomyelia (SM; CM-I+SM) are not well established.

METHODS Using the Park-Reeves Syringomyelia Research Consortium registry, the authors analyzed variables associated with syrinx radiological outcomes in patients (<20 years old at the time of surgery) with CM-I+SM undergoing PFD or PFDD. Syrinx resolution was defined as an anteroposterior (AP) diameter of $\leq 2 \mathrm{~mm}$ or $\leq 3 \mathrm{~mm}$ or a reduction in AP diameter of $\geq 50 \%$. Syrinx regression or progression was defined using 1) change in syrinx AP diameter ( $\geq 1 \mathrm{~mm})$, or 2) change in syrinx length (craniocaudal, $\geq 1$ vertebral level). Syrinx stability was defined as a $<1$-mm change in syrinx AP diameter and no change in syrinx length.

RESULTS The authors identified 380 patients with CM-I+SM who underwent PFD or PFDD. Cox proportional hazards modeling revealed younger age at surgery and PFDD as being independently associated with syrinx resolution, defined as a $\leq 2-\mathrm{mm}$ or $\leq 3-\mathrm{mm}$ AP diameter or $\geq 50 \%$ reduction in AP diameter. Radiological syrinx resolution was associated with improvement in headache $(p<0.005)$ and neck pain $(p<0.011)$ after PFD or PFDD. Next, PFDD $(p=0.005)$, scoliosis $(p=0.007)$, and syrinx location across multiple spinal segments $(p=0.001)$ were associated with syrinx diameter regression, whereas increased preoperative frontal-occipital horn ratio (FOHR; $p=0.007$ ) and syrinx location spanning multiple spinal segments $(p=0.04)$ were associated with syrinx length regression. Scoliosis (HR $0.38[95 \% \mathrm{Cl} 0.16-$ 0.91], $p=0.03$ ) and smaller syrinx diameter (5.82 \pm 3.38 vs $7.86 \pm 3.05 \mathrm{~mm} ; \mathrm{HR} 0.60$ [95\% Cl 0.34-1.03], $p=0.002)$ were associated with syrinx diameter stability, whereas shorter preoperative syrinx length $(5.75 \pm 4.01$ vs $9.65 \pm 4.31$ levels; HR 0.21 [95\% Cl 0.12-0.38], $p=0.0001)$ and smaller pB-C2 distance ( $6.86 \pm 1.27$ vs $7.18 \pm 1.38 \mathrm{~mm}$; HR 1.44 $[95 \% \mathrm{Cl} 1.02-2.05], p=0.04)$ were associated with syrinx length stability. Finally, younger age at surgery $(8.19 \pm 5.02$ vs $10.29 \pm 4.25$ years; HR 1.89 [95\% Cl 1.31-3.04], $p=0.01$ ) was associated with syrinx diameter progression, whereas increased postoperative syrinx diameter (6.73 \pm 3.64 vs $3.97 \pm 3.07 \mathrm{~mm}$; HR 3.10 [95\% Cl 1.67-5.76], $p=0.003)$, was associated with syrinx length progression. PFD versus PFDD was not associated with syrinx progression or reoperation rate.

CONCLUSIONS These data suggest that PFDD and age are independently associated with radiological syrinx improvement, although forthcoming results from the PFDD versus PFD randomized controlled trial (NCT02669836, clinicaltrials. gov) will best answer this question.

https://thejns.org/doi/abs/10.3171/2020.1.PEDS19493

KEYWORDS Chiari malformation type I; syrinx; Park-Reeves Syringomyelia Research Consortium

$\mathrm{C}$

HIARI malformation type I (CM-I) is usually defined as displacement of the cerebellar tonsils $\geq 5$ $\mathrm{mm}$ below the foramen magnum, with clinical presentation ranging from asymptomatic to severe occipitocervical headaches and diminished quality of life. ${ }^{21} \mathrm{CM}-\mathrm{I}$ is commonly associated with syringomyelia (SM), ${ }^{18,26}$ a fluid-filled cavity within the spinal cord, which, if left untreated, can lead to severe neurological impairment. The primary goal of surgical treatment for patients with CMI with SM (CM-I+SM) is to expand the posterior fossa space, presumably attenuating the craniospinal pressure dissociation, relieving CSF obstruction, and decreasing the syrinx size. However, demographic, clinical, radiological, and surgical factors associated with postoperative syrinx size are not well defined. In addition, radiological definitions of syrinx improvement vary widely in the literature, and prior meta-analyses have yielded mixed findings. ${ }^{9} 12,23,25,30$ Thus, there is a need for a systematic, multiinstitutional study of pediatric patients undergoing operative treatment for CM-I+SM and analysis of multiple covariates that may influence radiological syrinx improvement or progression of disease.

The goal of our study was to identify demographic, clinical, radiological, and surgical factors associated with 
syrinx size in pediatric patients with CM-I+SM undergoing posterior fossa decompression (PFD) or PFD with duraplasty (PFDD) using the retrospective component of the multiinstitutional Park-Reeves Syringomyelia Research Consortium (PRSRC) database. Since radiological syrinx improvement can be, and has been, defined in many ways, we considered radiological syrinx outcomes in multiple ways: 1) syrinx resolution (postoperative syrinx anteroposterior [AP] diameter $\leq 2 \mathrm{~mm}$ as a dichotomous variable or $\leq 3 \mathrm{~mm}$ as a secondary dichotomous outcome); 2) syrinx resolution, where $\geq 50 \%$ reduction in AP diameter was treated as a dichotomous variable; 3) syrinx regression, where any reduction in AP diameter $\geq 1 \mathrm{~mm}$ was treated as a continuous variable; 4) syrinx length regression (i.e., reduction in the number of craniocaudal spinal levels occupied by the syrinx, as a continuous variable); 5) syrinx stability, defined as a $<1$-mm change in postoperative AP diameter or $<1$ vertebral level change in syrinx length; and 6) syrinx progression, defined as an increase in postoperative syrinx diameter or as an increase in syrinx length of $\geq 1$ spinal level (dichotomous variables). These data provide a cohesive, multiinstitutional analysis of factors associated with radiological syrinx outcomes in pediatric patients undergoing PFD or PFDD for CM-I+SM.

\section{Methods \\ Study Design}

We utilized the retrospectively collected component of the ambispective PRSRC patient registry, which enrolls patients from 42 institutions across North America. We included all patients with CM-I+SM younger than 20 years at surgery undergoing PFD or PFDD between 2001 and 2015 regardless of syrinx location or size. Only patients undergoing their first PFD or PFDD who were followed up for a minimum of 6 months were included. Our primary outcome was syrinx resolution, defined as a maximal width of $\leq 2 \mathrm{~mm}$ in the AP direction on postoperative sagittal T2-weighted MRI. Thus, we dichotomized our cohort into patients whose syrinx resolved and those whose syrinx did not at the last available radiological evaluation. Secondary analyses included syrinx resolution $(\leq$ 3-mm AP diameter), syrinx resolution ( $\geq 50 \%$ reduction in AP diameter), syrinx diameter regression (AP diameter treated as a continuous variable), and syrinx length regression (craniocaudal, spinal levels occupied treated as a continuous variable). Next, we identified factors associated with syrinx stability, defined separately as 1) no change in postoperative syrinx diameter or 2) no change in syrinx length after PFD or PFDD. Finally, we analyzed factors associated with syrinx progression, defined separately as an increase in 1) AP syrinx diameter or 2) syrinx length after PFD or PFDD. The threshold for syrinx regression or progression was $1 \mathrm{~mm}$ in AP diameter or 1 vertebral level in craniocaudal length. Institutional review board approval was obtained at all participating sites. All surgical and clinical management decisions were at the sole discretion of the surgeon(s) at each clinical site. Likewise, imaging was performed at the discretion of the clinical team at each site and was not standardized. All MRI measurements were made by a central reader and confirmed by a board-certified neuroradiologist. All analyses were performed blinded to institution and surgeon.

\section{Data Collection}

We analyzed the following demographic characteristics: 1) age at PFD or PFDD for CM-I+SM, 2) sex, and 3) race (Caucasian vs non-Caucasian). We also analyzed neurological disease history, including 1) history of congenital brain malformation (Dandy-Walker malformation, encephalocele, malformation of cortical development, and other brain malformations), 2) scoliosis as indicated in the medical history, 3) kyphosis as indicated in the medical history, 4) skull base malformation, 5) platybasia, 6) basilar invagination, 7) retroflexion of the dens, and 8) history of hydrocephalus treated with permanent CSF diversion prior to PFD or PFDD. The following radiological variables were collected at each individual clinical site: 1) distance from the ventral dura perpendicular to the line between the basion and $\mathrm{C} 2$ (pB-C2 distance), 2) degree of tonsillar ectopia (mm), 3) clivus canal angle (CXA), 4) preoperative frontal-occipital horn ratio (FOHR), and 5) pre- and postoperative syrinx diameter. Surgical and intraoperative variables included PFD versus PFDD, intradural maneuvers (arachnoid sparing, intradural lysis of adhesions, or tonsillar reduction), intraoperative fenestration of the syrinx, or syrinx shunt placement.

\section{Statistical Analysis}

The chi-square test was used to compare two categorical variables, and the Wilcoxon rank-sum test was used to compare two continuous variables assumed to not be normally distributed. Multivariate linear regression was used to analyze syrinx diameter and syrinx length regression. We used the risk-adjusted Cox proportional hazards $(\mathrm{CPH})$ modeling using time after PFD or PFDD to earliest follow-up with maximal syrinx regression or progression as the time variable and syrinx resolution as the event. All multivariate analyses included any variable reaching statistical significance ( $\mathrm{p}<0.05$, defined a priori) by univariate analysis as covariates. A highly conservative Bonferroni correction was performed to adjust for the number of statistical tests performed in each model. No data imputation was performed for any missing data.

\section{Results \\ Cohort Description}

Female patients accounted for $60 \%$ of the cohort (Table 1). The mean age at diagnosis was $10.05 \pm 4.40$ years, and the mean follow-up was $1.55 \pm 0.06$ years. Our primary outcome of interest was syrinx resolution $(\leq 2 \mathrm{~mm}$ in the AP plane after PFD or PFDD), where 145 patients $(38 \%)$ experienced complete syrinx resolution. On univariate analysis, we found that female sex (43\% vs $31 \%, \mathrm{p}=0.014)$, younger age at surgery (treated as a categorical variable, $p$ $=0.003)$, and increased length of follow-up $(1.73 \pm 0.04 \mathrm{vs}$ $1.44 \pm 0.08$ years, $\mathrm{p}=0.018$ ) were associated with syrinx resolution. We then considered neurological comorbidities and found that none of the following diagnoses were associated with syrinx resolution: brain malformation, basilar invagination, skull base abnormalities, scoliosis, 
kyphosis, platybasia, or retroflexion of the dens. Next, we investigated radiological variables and their association with syrinx resolution and found that smaller preoperative syrinx diameter $(6.56 \pm 2.89$ vs $8.33 \pm 3.10 \mathrm{~mm}, \mathrm{p}<$ $0.001)$ and decreased postoperative syrinx length (5.99 \pm 3.89 vs $8.01 \pm 4.59$ spinal levels occupied by the syrinx, $p$ $<0.001$ ), but not preoperative syrinx length, were associated with syrinx resolution. The following variables were not associated with syrinx resolution: $\mathrm{pB}-\mathrm{C} 2$ distance, tonsillar descent, CXA, preoperative FOHR, history of hydrocephalus, history of permanent CSF diversion, or syrinx location. Lastly, we considered operative variables associated with syrinx resolution and found that PFDD $(86 \%)$ vs PFD $(77 \%, p=0.028)$, but not intradural maneuvers (arachnoid sparing, intradural lysis of adhesions, or tonsillar reduction), fenestration of syrinx, or placement of a syrinx shunt, was associated with syrinx resolution (Table 1).

\section{Factors Associated With Syrinx Resolution by Multivariate and Time-to-Event Analysis}

Since increased follow-up time was associated with syrinx resolution $(1.73 \pm 0.04$ vs $1.44 \pm 0.08$ years, $p=$ 0.018 ), we constructed a CPH model to analyze syrinx resolution $(\leq 2 \mathrm{~mm})$ using the following covariates: 1) age at surgery (treated as a categorical variable, 0 to $<5,5$ to $<$ 10,10 to $<15$, and 15 to $<20$ years), 2) sex, 3) PFDD, and 4) preoperative syrinx diameter (treated as a categorical variable, 0 to $5 \mathrm{~mm},>5$ to $\leq 10 \mathrm{~mm}$, and $>10 \mathrm{~mm}$ ). The earliest time when the maximal amount of regression was observed (i.e., smallest postoperative syrinx diameter) was defined as time. Using this method, we identified younger age at surgery (HR 1.10 [95\% CI 1.04-1.16], $\mathrm{p}=0.0002$ ) and PFDD (HR 1.78 [95\% CI 1.00-3.17], $\mathrm{p}=0.049$ ), but not preoperative syrinx diameter or sex, as independently associated with syrinx resolution (Table 2). Of note, younger age at surgery remained statistically significant after a highly conservative Bonferroni correction ( $\mathrm{p}_{\text {Bonfer- }}$ roni) for total number of variables tested.

We also considered a less-stringent definition of syrinx resolution $(\leq 3-\mathrm{mm}$ AP diameter, $\mathrm{n}=198)$ and found a similar relationship between PFDD (HR 1.89 [95\% CI 1.22-2.07], $\mathrm{p}=0.014)$ and age (HR 1.21 [95\% CI 1.11$1.28], \mathrm{p}=0.001$ ) and syrinx resolution (Table 2). Furthermore, we considered variables associated with $\geq 50 \%$ reduction in syrinx diameter after PFD or PFDD $(n=190)$. We recapitulated the findings above and found that PFDD (HR 2.04 [95\% CI 1.34-2.69], $\mathrm{p}=0.039$ ) and younger age at surgery (HR 1.08 [95\% CI 1.01-1.22], $\mathrm{p}=0.001$ ) were associated with $\mathrm{a} \geq 50 \%$ reduction in syrinx diameter (Table 2).

Our results suggest that syrinx resolution is quicker in patients undergoing PFDD, although most patients undergoing PFD also experienced syrinx resolution $(p=0.025$; Fig. 1). In addition, younger patients at surgery were far more likely to achieve faster and higher rates of syrinx resolution ( $p<0.001$; Fig. 2). Furthermore, we considered the association of radiological syrinx resolution $(\leq 2-\mathrm{mm}$ AP diameter) and improvement in headache and neck pain. Of the patients who experienced headaches preoperatively (71\%), those who achieved syrinx resolution were more likely to experience improvement in headache symptoms after PFD or PFDD than patients who did not experience syrinx resolution $(91 \%$ vs $77 \%, p<0.005)$. Similar rates of improvement in neck pain were observed in patients experiencing post-PFD or post-PFDD syrinx resolution $(86 \%$ vs $75 \%, \mathrm{p}<0.011)$. Thus, our data suggest that radiological syrinx improvement is similarly associated with improvement in clinical symptoms.

\section{Factors Associated With Syrinx Resolution Based on Preoperative Syrinx Diameter}

We also constructed separate risk-adjusted CPH models as a function of preoperative syrinx diameter. In patients with a syrinx AP diameter $>10 \mathrm{~mm}$, PFDD (HR 3.29 [95\% CI 2.88-4.99], $\mathrm{p}=0.009$ ) and younger age at surgery (HR 1.60 [95\% CI 1.29-1.94], p = 0.001) were associated with syrinx resolution $\leq 2 \mathrm{~mm}$ (Table 2). Furthermore, PFDD (HR 1.79 [95\% CI 1.08-3.27], $\mathrm{p}=0.041$ ), younger age at surgery (HR 1.08 [95\% CI 1.05-1.11], $\mathrm{p}=$ 0.020 ), and female sex (HR 1.99 [95\% CI 0.89-2.88], $\mathrm{p}$ $=0.039$ ) were independently associated with syrinx resolution among those patients whose preoperative syrinx diameter was $>5 \mathrm{~mm}$ but $\leq 10 \mathrm{~mm}$. Finally, in patients whose preoperative syrinx diameter was $\leq 5 \mathrm{~mm}$, none of the following were associated with syrinx resolution: PFDD, age at surgery, scoliosis, tonsillar descent, clivus angle, or preoperative FOHR resolution (Table 2).

\section{Factors Associated With Syrinx Regression by Multivariate Analysis}

Since complete syrinx resolution is not the only criterion defining post-PFD or post-PFDD radiological success, we considered postoperative syrinx AP diameter as a continuous variable. Using multivariate linear regression, we identified PFDD $(p=0.005)$, scoliosis $(p=0.007)$, and syrinx location spanning multiple spinal segments (e.g., cervicothoracic, thoracolumbar, or holocord, $\mathrm{p}=0.001$ ), but not age at surgery or $\mathrm{pB}-\mathrm{C} 2$ distance, as associated with syrinx regression (Table 3). It should be noted that PFDD, scoliosis, and syrinx location remained statistically significant after Bonferroni $\left(\mathrm{p}_{\text {Bonferroni }}\right)$ correction, increasing confidence in these associations. We then considered syrinx regression as postoperative regression in syrinx length (craniocaudal, continuous variable) and identified increased preoperative FOHR $(\mathrm{p}=0.007)$ and syrinx location spanning multiple spinal segments $(p=0.04)$ as independently associated with syrinx length regression. However, PFDD, sex, and post-PFD or post-PFDD syrinx diameter did not remain statistically significant after controlling for confounding covariates. It should also be noted that spinal level syrinx regression, but not syrinx AP diameter, was positively and linearly associated with followup time $\left(\mathrm{p}=0.05, \mathrm{r}^{2}=0.72\right)$, suggesting that syringes may eventually resolve by this definition, although outside the time frame of data collected for this study.

\section{Factors Associated With Syrinx Stability by Multivariate Analysis}

Next, we considered patients who experienced no postoperative change in syrinx diameter at last avail- 
TABLE 1. Patient cohort characteristics and predictors of syrinx resolution $(\leq 2 \mathrm{~mm})$

\begin{tabular}{|c|c|c|c|c|}
\hline & No Syrinx Resolution $(n=235)$ & Syrinx Resolution ( $n=145$ ) & All Patients $(n=380)$ & p Value \\
\hline \multicolumn{5}{|l|}{ Demographics } \\
\hline Sex & & & & $0.014^{*}$ \\
\hline Male & $106(45)$ & $47(32)$ & $153(40)$ & \\
\hline Female & $129(55)$ & $98(68)$ & $227(60)$ & \\
\hline Race & & & & $0.268^{*}$ \\
\hline Non-Caucasian & $48(20)$ & $23(16)$ & $71(19)$ & \\
\hline Caucasian & $187(80)$ & $122(84)$ & 309 (81) & \\
\hline Age at surgery, yrs & & & & $0.003^{*}$ \\
\hline 15 to $<20$ & 32 & 22 & 54 & \\
\hline 10 to $<15$ & 60 & 83 & 143 & \\
\hline 5 to $<10$ & 30 & 85 & 115 & \\
\hline 0 to $<5$ & 13 & 45 & 58 & \\
\hline Mean follow-up time, yrs & $1.44 \pm 0.08$ & $1.73 \pm 0.04$ & $1.55 \pm 0.06$ & $0.018 \dagger$ \\
\hline \multicolumn{5}{|l|}{ Comorbidities } \\
\hline Brain malformation & & & & $0.052^{*}$ \\
\hline No & $229(97)$ & $145(100)$ & $374(98)$ & \\
\hline Yes & $6(3)$ & $0(0)$ & $6(2)$ & \\
\hline Basilar invagination & & & & $0.969^{*}$ \\
\hline No & $230(98)$ & $142(98)$ & $372(98)$ & \\
\hline Yes & $5(2)$ & $3(2)$ & $8(2)$ & \\
\hline Skull base abnormality & & & & $0.561^{*}$ \\
\hline No & $224(95)$ & $140(97)$ & $364(96)$ & \\
\hline Yes & $11(5)$ & $5(3)$ & $9(2)$ & \\
\hline Scoliosis (untreated) & & & & $0.704^{*}$ \\
\hline No & $138(59)$ & $88(61)$ & $226(59)$ & \\
\hline Yes & $97(41)$ & $57(39)$ & $154(41)$ & \\
\hline Kyphosis & & & & $0.319^{*}$ \\
\hline No & $228(97)$ & $143(99)$ & $371(98)$ & \\
\hline Yes & $7(3)$ & $2(1)$ & $9(2)$ & \\
\hline Platybasia & & & & $0.590^{*}$ \\
\hline No & $228(97)$ & $142(98)$ & $370(97)$ & \\
\hline Yes & $7(3)$ & $3(2)$ & $10(3)$ & \\
\hline Dens retroflexion & & & & $0.172^{*}$ \\
\hline No & $232(99)$ & $145(100)$ & 377 (99) & \\
\hline Yes & $3(1)$ & $0(0)$ & $3(1)$ & \\
\hline Immediate postop EVD & & & & $0.12^{*}$ \\
\hline No & $220(94)$ & $141(97)$ & $361(95)$ & \\
\hline Yes & $15(6)$ & $4(3)$ & $19(5)$ & \\
\hline VP shunt placement prior to PFD or PFDD & & & & $0.27^{*}$ \\
\hline No & $230(98)$ & $144(99)$ & $374(98)$ & \\
\hline Yes & $5(2)$ & $1(<1)$ & $6(2)$ & \\
\hline \multicolumn{5}{|l|}{ Imaging findings } \\
\hline $\mathrm{pB}-\mathrm{C} 2$ distance, $\mathrm{mm}$ & & & & $0.174^{*}$ \\
\hline 4 & $4(2)$ & $1(1)$ & $5(1)$ & \\
\hline 5 & 26 (11) & $22(15)$ & $48(13)$ & \\
\hline 6 & $63(27)$ & $24(17)$ & $87(23)$ & \\
\hline 7 & $77(33)$ & $44(30)$ & $121(32)$ & \\
\hline 8 & $45(19)$ & $38(26)$ & $83(22)$ & \\
\hline 9 & $13(6)$ & $9(6)$ & $22(6)$ & \\
\hline
\end{tabular}


Hale et al.

» CONTINUED FROM PAGE 633

TABLE 1. Patient cohort characteristics and predictors of syrinx resolution $(\leq 2 \mathrm{~mm})$

\begin{tabular}{|c|c|c|c|c|}
\hline & No Syrinx Resolution ( $\mathrm{n}=235$ ) & Syrinx Resolution ( $n=145)$ & All Patients $(n=380)$ & $p$ Value \\
\hline \multicolumn{5}{|l|}{ Imaging findings (continued) } \\
\hline pB-C2 distance, mm (continued) & & & & $0.174^{*}$ \\
\hline 10 & $6(3)$ & $7(5)$ & $13(3)$ & \\
\hline 12 & $1(<1)$ & $0(0)$ & $1(<1)$ & \\
\hline Mean tonsillar descent, mm & $12.92 \pm 4.92$ & $12.14 \pm 4.95$ & $12.64 \pm 4.94$ & $0.080 \dagger$ \\
\hline Mean CXA, ${ }^{\circ}$ & $144.2 \pm 12.3$ & $144.8 \pm 11.8$ & $144.4 \pm 12.1$ & $0.655 \dagger$ \\
\hline Mean FOHR & $0.307 \pm 0.040$ & $0.297 \pm 0.031$ & $0.303 \pm 0.037$ & $0.255 \dagger$ \\
\hline Mean no. of preop syrinx levels & $8.85 \pm 4.49$ & $8.82 \pm 4.64$ & $8.84 \pm 4.54$ & $0.871 \dagger$ \\
\hline Mean no. of postop syrinx levels & $8.01 \pm 4.59$ & $5.99 \pm 3.89$ & $7.25 \pm 4.45$ & $<0.001 \dagger$ \\
\hline Mean preop syrinx diameter, mm & $8.33 \pm 3.10$ & $6.56 \pm 2.89$ & $7.66 \pm 3.14$ & $<0.001 \dagger$ \\
\hline Mean postop syrinx diameter, $\mathrm{mm}$ & $6.29 \pm 3.09$ & $1.54 \pm 0.59$ & $4.48 \pm 3.37$ & $<0.001 \dagger$ \\
\hline Mean preop syrinx location & & & & $0.301^{*}$ \\
\hline Cervical & $42(22)$ & $31(27)$ & $73(24)$ & \\
\hline Thoracic & $21(11)$ & $8(7)$ & $29(10)$ & \\
\hline Holocord & $55(29)$ & $26(23)$ & $81(27)$ & \\
\hline Cervicothoracic & $72(38)$ & $42(47)$ & $119(39)$ & \\
\hline Cervicolumbar & $0(0)$ & $1(1)$ & $1(<1)$ & \\
\hline \multicolumn{5}{|l|}{ Surgical factors } \\
\hline PFDD & & & & $0.028^{*}$ \\
\hline No & $54(23)$ & $20(14)$ & $74(19)$ & \\
\hline Yes & $181(77)$ & $125(86)$ & $306(81)$ & \\
\hline Arachnoid sparing & & & & $0.51^{*}$ \\
\hline No & $221(94)$ & $133(92)$ & $354(93)$ & \\
\hline Yes & $14(6)$ & $12(8)$ & $26(7)$ & \\
\hline Tonsillar reduction & & & & $0.78^{*}$ \\
\hline No & $120(51)$ & & $197(52)$ & \\
\hline Yes & $115(49)$ & $77(53)$ & $183(48)$ & \\
\hline Intradural lysis of adhesions & & & & $0.39^{*}$ \\
\hline No & $159(68)$ & $91(63)$ & $250(66)$ & \\
\hline Yes & $76(32)$ & $54(37)$ & $130(34)$ & \\
\hline Syrinx shunt & & & & $0.275^{*}$ \\
\hline No & $230(98)$ & $144(99)$ & $374(98)$ & \\
\hline Yes & $5(2)$ & $1(1)$ & $6(2)$ & \\
\hline Fenestrated syrinx & & & & $0.265^{\star}$ \\
\hline No & $233(99)$ & $145(100)$ & $378(99)$ & \\
\hline Yes & $2(1)$ & $0(0)$ & 2 (1) & \\
\hline
\end{tabular}

Values represent the number of patients (\%) unless stated otherwise. Mean data are presented as mean \pm SEM.

${ }^{*}$ Chi-square test.

† Wilcoxon signed-rank test.

able follow-up $(\mathrm{n}=40)$, which we define as syrinx diameter stability. Univariate analysis identified preoperative syrinx diameter, scoliosis, PFDD, and preoperative syrinx levels as associated with syrinx diameter stability, prompting their inclusion as covariates in our model. Risk-adjusted CPH modeling revealed scoliosis (HR 0.38 [95\% CI 0.16-0.91], $\mathrm{p}=0.03$ ) and smaller syrinx diameter $(5.82 \pm 3.38$ vs $7.86 \pm 3.05 \mathrm{~mm}$; HR 0.60 [95\% CI $0.34-1.03], \mathrm{p}=0.002$ ) as predictors of syrinx stability
(Table 4). In addition, we conducted a subset analysis of patients who experienced no change in syrinx length after PFD or PFDD, which we define as syrinx length stability $(\mathrm{n}=75)$. Risk-adjusted CPH modeling identified smaller preoperative syrinx length $(5.75 \pm 4.01$ vs $9.65 \pm 4.31 \mathrm{lev}-$ els; HR 0.21 [95\% CI 0.12-0.38], $\mathrm{p}=0.0001$ ) and smaller pB-C2 distance $(6.86 \pm 1.27$ vs $7.18 \pm 1.38 \mathrm{~mm}$; HR 1.44 [95\% CI 1.02-2.05], $\mathrm{p}=0.04$ ) as associated with syrinx length stability (Table 4). 
TABLE 2. Factors associated with syrinx resolution, syrinx regression, or preoperative syrinx diameter by risk-adjusted $\mathrm{CPH}$ modeling using follow-up time as event

\begin{tabular}{|c|c|c|}
\hline & $\operatorname{HR}(95 \% \mathrm{Cl})$ & $\mathrm{p}$ Value \\
\hline \multicolumn{3}{|l|}{$\begin{array}{l}\text { Syrinx resolution ( } \leq 2-\mathrm{mm} \mathrm{AP} \\
\text { diameter) }\end{array}$} \\
\hline PFDD & $1.78(1.00-3.17)$ & 0.049 \\
\hline Younger age at surgery & $1.10(1.04-1.16)$ & 0.0002 \\
\hline Preop syrinx diameter & $0.84(0.68-1.23)$ & 0.081 \\
\hline Female sex & $1.76(1.13-2.75)$ & 0.157 \\
\hline \multicolumn{3}{|l|}{$\begin{array}{l}\text { Syrinx resolution }(\leq 3-\mathrm{mm} \mathrm{AP} \\
\text { diameter) }\end{array}$} \\
\hline PFDD & $1.89(1.22-2.07)$ & 0.014 \\
\hline Younger age at surgery & $1.21(1.11-1.28)$ & 0.001 \\
\hline Female sex & $1.01(0.47-2.46)$ & 0.320 \\
\hline \multicolumn{3}{|c|}{$\begin{array}{l}\text { Syrinx resolution ( } \geq 50 \% \text { decrease in } \\
\text { AP diameter) }\end{array}$} \\
\hline PFDD & $2.04(1.34-2.69)$ & 0.039 \\
\hline Younger age at surgery & $1.08(1.01-1.22)$ & 0.001 \\
\hline Scoliosis & $1.74(0.89-2.88)$ & 0.100 \\
\hline \multicolumn{3}{|c|}{$\begin{array}{l}\text { Syrinx resolution (AP diameter >10 } \\
\mathrm{mm} \text { ) }\end{array}$} \\
\hline PFDD & $3.29(2.88-4.99)$ & 0.009 \\
\hline Younger age at surgery & $1.60(1.29-1.94)$ & 0.001 \\
\hline \multicolumn{3}{|c|}{$\begin{array}{l}\text { Syrinx resolution (AP diameter }>5 \text { to } \\
\leq 10 \mathrm{~mm} \text { ) }\end{array}$} \\
\hline PFDD & $1.79(1.08-3.27)$ & 0.041 \\
\hline Younger age at surgery & $1.08(1.05-1.11)$ & 0.020 \\
\hline Female sex & $1.99(0.89-2.88)$ & 0.039 \\
\hline \multicolumn{3}{|c|}{$\begin{array}{l}\text { Syrinx resolution (AP diameter } \leq 5 \\
\mathrm{~mm} \text { ) }\end{array}$} \\
\hline Age at surgery & $1.07(0.98-1.17)$ & 0.457 \\
\hline Scoliosis & $1.87(0.69-5.09)$ & 0.549 \\
\hline Tonsillar descent & $0.85(0.75-0.93)$ & 0.079 \\
\hline Clivus angle & $0.99(0.96-1.02)$ & 0.753 \\
\hline FOHR & $0.47(0.32-0.65)$ & 0.082 \\
\hline
\end{tabular}

\section{Factors Associated With Syrinx Progression by Multivariate Analysis}

We then considered patients who experienced an increase in post-PFD or post-PFDD syrinx diameter at a minimum of 6 months of follow-up, which was defined here as syrinx diameter progression $(n=44)$. We constructed a risk-adjusted CPH model and identified younger age at surgery $(8.19 \pm 5.02$ vs $10.29 \pm 4.25$ years; HR 1.89 [95\% CI 1.31-3.04], $\mathrm{p}=0.01$ ) and increased preoperative CXA $\left(148.5^{\circ} \pm 12.5^{\circ}\right.$ vs $143.9^{\circ} \pm 12.0^{\circ}$; HR $1.53[95 \%$ CI $0.99-2.38], p=0.05$; Table 5) as associated with syrinx progression. Of note, 18 patients $(41 \%)$ who experienced syrinx progression were $<5$ years old, $8(18 \%)$ were 5 to $<$ 10 years old, $14(32 \%)$ were 10 to $<15$ years old, and $4(9 \%)$ were 15 to $<20$ years old $\left(\mathrm{p}=1 \times 10^{-5}\right.$ by chi-square test, significant after Bonferroni correction). However, when preoperative CXA was treated as a categorical variable

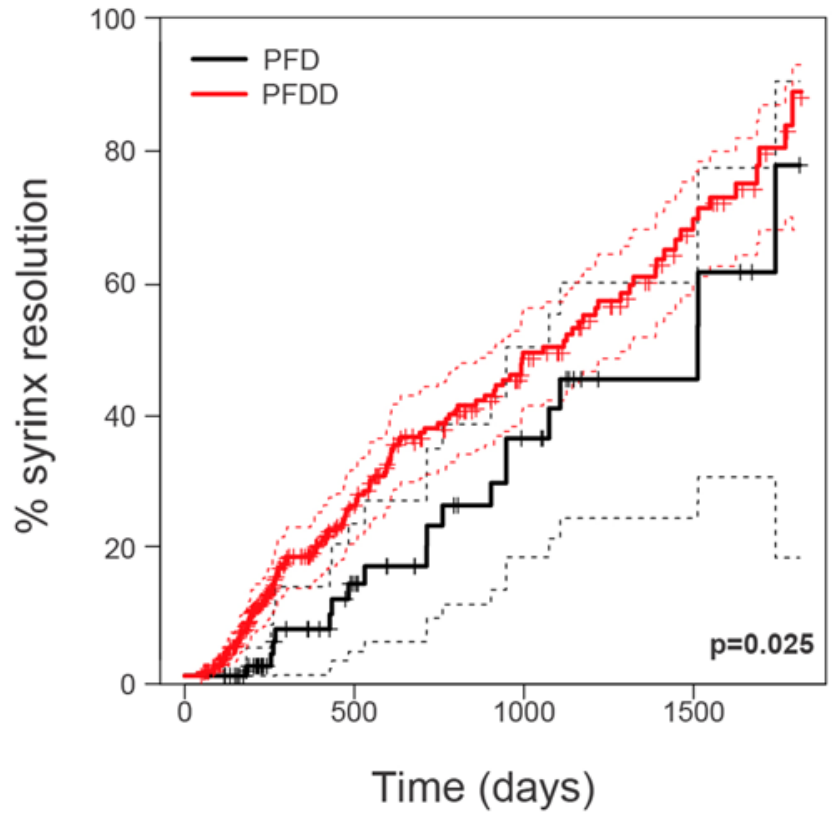

FIG. 1. Cox proportional hazards analysis of patients achieving syrinx resolution (i.e., event) over time as a function of PFDD (red line) versus PFD (black line). The dashed lines represent the 95\% Cl. Figure is available in color online only.

$\left(\mathrm{CXA} \leq 125^{\circ}, 125^{\circ}<\mathrm{CXA} \leq 135^{\circ}, 135^{\circ}<\mathrm{CXA} \leq 145^{\circ}\right.$, $145^{\circ}<\mathrm{CXA} \leq 155^{\circ}$, and $\left.\mathrm{CXA}>155^{\circ}\right)$ there was no association with syrinx progression ( $\mathrm{p}=0.09$, chi-square test). Thus, while CXA (continuous variable) was independently associated with syrinx progression, whether or not this is a clinically relevant distinction remains unclear. Finally, we identified greater postoperative syrinx AP diameter (6.73

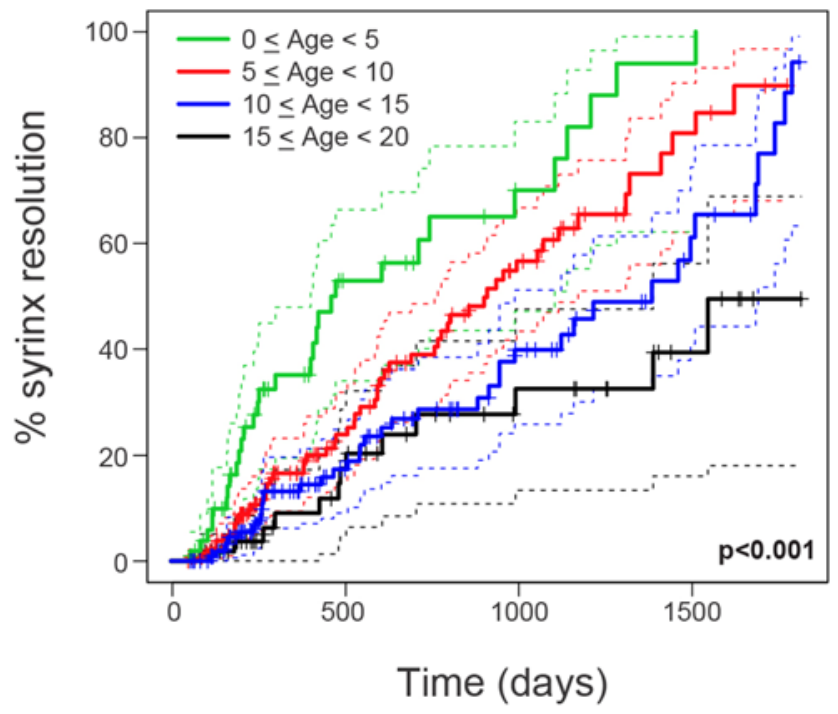

FIG. 2. Cox proportional hazards analysis of patients achieving syrinx resolution (i.e., event) over time as a function of age at surgery (years). The dashed lines represent the $95 \% \mathrm{Cl}$. Figure is available in color online only. 
TABLE 3. Factors associated with syrinx regression (continuous) by linear regression

\begin{tabular}{lccc}
\hline \multicolumn{1}{c}{ Variable } & Coefficient & SE & p Value \\
\hline AP diameter & & & \\
\hline PFDD & -2.45 & 0.86 & 0.005 \\
\hline Scoliosis & -1.16 & 0.34 & 0.007 \\
\hline Syrinx location & -0.32 & 0.10 & 0.001 \\
\hline Age at surgery & 0.10 & 0.10 & 0.37 \\
\hline pB-C2 distance & -0.04 & 0.12 & 0.70 \\
\hline Craniocaudal length & & & \\
\hline Preop FOHR & 22.18 & 8.04 & 0.007 \\
\hline Syrinx location & -0.38 & 0.19 & 0.04 \\
\hline Follow-up time & -0.001 & 0.007 & 0.05 \\
\hline PFDD & -1.49 & 1.37 & 0.28 \\
\hline Sex & 0.87 & 0.64 & 0.17 \\
\hline Postop syrinx diameter & 0.25 & 0.32 & 0.42 \\
\hline
\end{tabular}

\pm 3.64 vs $3.97 \pm 3.07 \mathrm{~mm}$; HR 3.10 [95\% CI 1.67-5.76], p $=0.003)$, but not PFD or PFDD, as associated with syrinx length progression $(n=74)$ (Table 5). Of note, there was no difference in reoperation rate between patients undergoing PFD versus PFDD, and PFD versus PFDD was not associated with syrinx diameter or syrinx length progression.

\section{Discussion}

This report describes one of the largest multiinstitutional case series of pediatric patients undergoing PFD or PFDD for treatment of CM-I+SM. Our central finding is that PFDD and younger age remained significantly associated with improved radiological syrinx improvement using both strict (i.e., $\leq 2$ - or $\leq 3$-mm resolution) and lessstringent (i.e., reduction in syrinx AP diameter $\geq 50 \%$ ) definitions of syrinx improvement. Patients with scoliosis and syrinx occupying multiple spinal levels were more likely to experience syrinx diameter regression. Interestingly, patients with scoliosis and smaller syrinx diameter were less likely to experience syrinx diameter stability,

TABLE 4. Factors associated with syrinx stability using risk-adjusted $\mathrm{CPH}$ modeling

\begin{tabular}{lll}
\hline \multicolumn{1}{c}{ Variable } & HR $(95 \% \mathrm{Cl})$ & $\mathrm{p} \mathrm{Value}$ \\
\hline AP syrinx diameter stability & & \\
\hline Preop syrinx diameter & $0.60(0.34-1.03)$ & 0.002 \\
\hline Scoliosis & $0.38(0.16-0.91)$ & 0.03 \\
\hline PFDD & $0.55(0.28-1.09)$ & 0.09 \\
\hline Preop syrinx length (craniocaudal) & $0.81(0.45-1.45)$ & 0.48 \\
\hline Syrinx level (craniocaudal) stability & & \\
\hline Preop syrinx levels & $0.21(0.12-0.38)$ & 0.0001 \\
\hline pB-C2 distance & $1.44(1.02-2.05)$ & 0.04 \\
\hline Preop syrinx diameter & $0.98(0.66-1.47)$ & 0.11 \\
\hline Scoliosis & $0.86(0.51-1.47)$ & 0.58 \\
\hline
\end{tabular}

TABLE 5. Factors associated with syrinx progression using risk-adjusted $\mathrm{CPH}$ modeling

\begin{tabular}{lll}
\hline \multicolumn{1}{c}{ Variable } & $\mathrm{HR}(95 \% \mathrm{Cl})$ & $\mathrm{p} \mathrm{Value}$ \\
\hline AP syrinx diameter progression & & \\
\hline Age at surgery & $1.89(1.31-3.04)$ & 0.01 \\
\hline Mean CXA & $1.53(0.99-2.38)$ & 0.05 \\
\hline pB-C2 & $0.97(0.73-1.28)$ & 0.82 \\
\hline Basilar invagination & $3.20(0.52-19.87)$ & 0.21 \\
\hline Skull base abnormality & $3.65(0.81-16.56)$ & 0.09 \\
\hline Syrinx level (craniocaudal) progression & & \\
\hline Postop syrinx diameter & $3.10(1.67-5.76)$ & 0.003 \\
\hline PFD & $0.97(0.57-1.63)$ & 0.89 \\
\hline
\end{tabular}

whereas fewer preoperative syrinx levels and smaller $\mathrm{pB}$ C2 distance were associated with syrinx length stability. Younger age at surgery and patients with a larger preoperative CXA were more likely to experience syrinx diameter progression, and patients with larger postoperative syrinx diameter were more likely to experience syrinx length progression. Younger patients at surgery were also more likely to experience syrinx resolution or progression, suggesting that younger patients are more sensitive to PFD or PFDD. Notably, there was no difference in reoperation rate or syrinx progression between patients undergoing PFD versus PFDD.

Reported rates of syrinx improvement in pediatric patients undergoing PFD or PFDD vary widely. This is likely due to inconsistent definitions of syrinx improvement, the presence of uncontrolled comorbidities, differences in operative technique, and the inclusion of case series with small sample sizes (including single-center/surgeon studies), among others. ${ }^{1-4,24}$ Syrinx improvement is defined inconsistently in the literature, and discrepancies are raised from pooling heterogeneous studies with varying definitions of radiological syrinx improvement. To overcome these limitations and enable comparison of our study with other series, we 1) analyzed syrinx resolution, regression, stability, and progression separately using a data-driven approach (i.e., performed multivariate analysis using only those variables reaching statistical significance by univariate analysis, where we did not encounter issues related to statistical degrees of freedom, thus mitigating the risk of overfitting the statistical model); 2) defined syrinx regression and progression in a number of ways; and, finally, 3) included all patients with CM-I+SM undergoing PFD or PFDD regardless of comorbidities over a wide time frame (2001-2015), where practice patterns differed across institutions and surgeons. Furthermore, because nuances in operative technique and surgeon-specific heuristic differences could not be captured in the PRSRC registry, our approach was purposefully designed to identify generalizable surgical approaches and risk factors associated with objective, radiological post-PFD or post-PFDD CM-I+SM syrinx outcomes.

Perhaps the most-debated question in treating patients with $\mathrm{CM}-\mathrm{I}+\mathrm{SM}$ is whether or not to combine PFD with duraplasty. ${ }^{5}$ A number of meta-analyses have been 
performed to answer this question; however, differing conclusions have been reached, largely due to the issues raised above..$^{9,12,16,23}$ Among other considerations, the PFD versus PFDD debate prompted a randomized controlled trial (NCT02669836, clinicaltrials.gov) to provide level I evidence for this important question. While the results of the randomized controlled trial will provide the strongest data comparing PFD and PFDD, in the interim we have analyzed retrospectively collected data from 380 patients from the multiinstitutional PRSRC network, a group of 42 institutions with one or more surgeons contributing patient-level data. These data agnostically identified PFDD (out of 23 variables tested) as being associated with both syrinx resolution (by 3 separate definitions) and regression using stringent multivariate analyses, providing supportive evidence for the utility of PFDD in syrinx resolution. Notably, however, patients undergoing PFD still achieved syrinx resolution, albeit at lesser frequency. In addition, intradural operative variables, including arachnoid sparing, intradural lysis of adhesions, and tonsillar reduction, were not associated with radiological syrinx improvement, consistent with prior studies. ${ }^{19}$ Furthermore, those patients who received PFDD experienced syrinx resolution faster than patients undergoing PFD (50\% syrinx resolution within approximately 2.7 years vs approximately 4.2 years). Since follow-up time was not standardized, this study also potentially serves as a useful reference for understanding the natural history of surgically treated CMI+SM resolution as a function of PFD or PFDD and age (Figs. 1 and 2).

Many studies have investigated the relationship between spinal deformity and CM-I+SM or CM-I+SM-related outcomes. ${ }^{13,15,17,20}$ However, these studies were predominantly focused on outcomes related to spinal deformity after PFD or PFDD, rather than measures of syrinx improvement. One study included 44 pediatric patients undergoing PFDD for CM-I+SM and scoliosis and reported syrinx improvement in $82 \%$ of patients by 6 months after PFD over the course of follow-up for this study. ${ }^{29}$ However, these authors defined syrinx resolution as a $>20 \%$ decrease in postoperative syrinx diameter, a much lessstringent criterion than that included here. We found that patients with scoliosis were more likely to experience syrinx regression, suggesting perhaps that these individuals have asymptomatic syringes that are only identified due to their scoliosis workup and thus identified earlier, consistent with our observation that younger patients experience syrinx resolution at higher rates. However, the relationship between CM-I, SM, and scoliosis is complex, and others have suggested that SM (but likely not CM-I) is independently associated with scoliosis. ${ }^{27}$ Thus, any relationship between CM-I, SM, and scoliosis should be interpreted with caution and not necessarily be considered causal.

In addition, we identify factors associated with syrinx length (craniocaudal) regression. We find that increased FOHR ( $p=0.007)$ and syrinx location spanning multiple spinal segments $(\mathrm{p}=0.04)$ remain statistically significant using multivariate linear regression. Here, we found that patients with increased ventricular size, as determined by FOHR and perhaps related to syrinx severity, ${ }^{28}$ are more likely to experience attenuation of decreased syrinx length. Consistent with this interpretation, patients with a longer preoperative syrinx length are more likely to experience a decrease in syrinx length after PFD or PFDD. However, these results did not apply to syrinx diameter regression, suggesting a different pathophysiological mechanism driving changes in syrinx diameter, or alternatively a relative lack of sensitivity for changes to this variable in the present study. Nonetheless, since one of the goals of PFD or PFDD is to attenuate CSF obstruction and improve craniocaudal flow, the present results suggest that patients with ventriculomegaly and longer syringes (craniocaudal) are more most likely to respond radiologically to PFD or PFDD treatment.

We then considered syrinx stability in a subgroup analysis, as these cases may be considered an operative success if the patient's symptoms and/or ventriculomegaly resolve, or conversely, unsuccessful if symptoms persist. Interestingly, we identified patients with smaller syrinx diameter and scoliosis as less likely to experience syrinx stability. Conversely, syrinx diameter has previously been associated with varying rates of syrinx improvement, ${ }^{8,10-12}$, $14,22,31$ ranging from approximately $50 \%$ to $100 \%$, making any definite claims about syrinx diameter and degree of regression and/or resolution difficult to interpret. In addition, we considered syrinx length stability in a separate subgroup analysis and identify shorter syringes in length at the time of PFD or PFDD as far less likely to experience syrinx stability (HR 0.21 [95\% CI 0.12-0.38]); i.e., longer syringes are more likely to persist after PFD or PFDD. Decreased pB-C2 distance was positively associated with syrinx length stability, although the magnitude of this change was small $(6.86 \pm 1.27$ vs $7.18 \pm 1.38 \mathrm{~mm})$, and the $\mathrm{pB}-\mathrm{C} 2$ distance has been shown to increase throughout development ${ }^{6}$ which may limit its utility as a prognostic metric.

Finally, we identified patients with an increased preoperative CXA as more likely to experience syrinx diameter progression. The nature of this relationship is unclear; a more acute clivus angle $\left(<125^{\circ}\right)$ has been considered a metric of craniovertebral junction instability in patients with CM-I, as these patients are more likely to require occipitocervical fusion. ${ }^{7}$ Thus, one might predict that a smaller clivus angle would be associated with syrinx progression; however, this hypothesis was not confirmed by these data. Importantly, when CXA was treated as a categorical variable, there was no association with syrinx diameter progression by univariate analysis. In addition, others have reported that clivus angle does not appear to correlate with syrinx diameter, ${ }^{15}$ consistent with our study's findings, suggesting that clivus angle is not necessarily a proxy marker for syrinx severity. However, the normative craniovertebral junction anatomy changes considerably throughout development, ${ }^{6}$ making age an important consideration. To complicate interpretation of this result, we identify younger age at surgery as associated with syrinx progression ( $8.19 \pm 5.02$ vs $10.29 \pm 4.25$ years), although there is a considerably large standard error, and these results are largely driven by $41 \%$ of patients experiencing syrinx progression who were younger than 5 years at surgery. Finally, and paradoxically, younger age at surgery is independently associated with both syrinx resolution and syrinx progression (AP diameter and length), although this 
may be driven by a greater percentage of patients younger than 5 years with larger syringes. These results suggest that, in younger patients, $\mathrm{CM}-\mathrm{I}+\mathrm{SM}$ is more sensitive to alteration of the underlying pathophysiology, such that surgery may result in either disease regression or, alternatively (presumably in the case of inadequate anatomical results of the operation), further progression.

It is also important to note that PFD versus PFDD was not associated with syrinx progression or need for reoperation. Coupled with the observation that PFDD was independently associated with syrinx resolution and regression, these data support a role for PFDD, although additional studies are warranted.

\section{Limitations}

This study was limited by retrospective data collection from 42 centers across North America since 2001, representing significant variation in the management of $\mathrm{CM}-\mathrm{I}+\mathrm{SM}$ between centers and over time. Furthermore, many nuances in operative technique and surgeon-specific approaches could not be captured using these data. Importantly, the decision to perform PFD versus PFDD was surgeon dependent, PFDD-specific approaches (i.e., arachnoid opening or the presence of a fourth ventricular web) were not standardized, and follow-up time was not standardized across institutions. Until 2016 with the creation of the Chiari Health Index for Pediatrics (CHIP), ${ }^{21}$ there was no disease-specific, standardized metric for quantifying patient-reported outcomes for pediatric patients with CM-I. Since the creation of this instrument, the PRSRC has collected CHIP data and will include these data in reports of the prospective PRSRC cohort and the ongoing randomized controlled trial for PFD or PFDD. Although recent meta-analyses have reported that undergoing PFDD is associated with symptom improvement in patients with CM-I+SM, clinical improvement may not be directly correlated with imaging-based metrics of syrinx reduction. ${ }^{23,25}$ Finally, primary imaging related to spinal deformity was only available for a subset of patients, precluding any detailed analysis of spinal column parameters before or after PFD or PFDD.

\section{Conclusions}

Our data provide evidence that PFDD can offer significant radiological improvement in syrinx resolution and regression. In addition, we found that younger patients at surgery are more likely to respond positively and resolve their syrinx or, in some cases, experience syrinx progression. We also provide data on a number of factors associated with craniocaudal syrinx regression, stability, and progression. Our study documents the positive influence of PFDD and young age on the reduction of SM in patients with CM-I+SM. A forthcoming randomized controlled trial will provide additional data on the utility of PFD versus PFDD for CM-I for clinical, radiological, and qualityof-life outcome measures.

\section{Acknowledgments}

We would like to thank the Reeves and Spears families for their generosity in establishing and supporting the Park-Reeves
Syringomyelia Research Consortium, Tammy Bethel-Anderson for her invaluable administrative support, and Alexander T. Yahanda for his assistance in data management. Dr. Hale is supported by the National Institutes of Health (F30HL143826) and Vanderbilt University Medical Scientist Training Program (5T32GM007347).

\section{References}

1. Alzate JC, Kothbauer KF, Jallo GI, Epstein FJ: Treatment of Chiari I malformation in patients with and without syringomyelia: a consecutive series of 66 cases. Neurosurg Focus 11(1):E3, 2001

2. Anderson RC, Dowling KC, Feldstein NA, Emerson RG: Chiari I malformation: potential role for intraoperative electrophysiologic monitoring. J Clin Neurophysiol 20:65-72, 2003

3. Anderson RC, Emerson RG, Dowling KC, Feldstein NA: Improvement in brainstem auditory evoked potentials after suboccipital decompression in patients with Chiari I malformations. J Neurosurg 98:459-464, 2003

4. Attenello FJ, McGirt MJ, Gathinji M, Datoo G, Atiba A, Weingart J, et al: Outcome of Chiari-associated syringomyelia after hindbrain decompression in children: analysis of 49 consecutive cases. Neurosurgery 62:1307-1313, 2008

5. Baisden J: Controversies in Chiari I malformations. Surg Neurol Int 3 (Suppl 3):S232-S237, 2012

6. Bapuraj JR, Bruzek AK, Tarpeh JK, Pelissier L, Garton HJL, Anderson RCE, et al: Morphometric changes at the craniocervical junction during childhood. J Neurosurg Pediatr 24:227-235, 2019

7. Bollo RJ, Riva-Cambrin J, Brockmeyer MM, Brockmeyer DL: Complex Chiari malformations in children: an analysis of preoperative risk factors for occipitocervical fusion. $\mathbf{J}$ Neurosurg Pediatr 10:134-141, 2012

8. Caldarelli M, Novegno F, Vassimi L, Romani R, Tamburrini G, Di Rocco C: The role of limited posterior fossa craniectomy in the surgical treatment of Chiari malformation type I: experience with a pediatric series. J Neurosurg 106 (3 Suppl):187-195, 2007

9. Chai Z, Xue X, Fan H, Sun L, Cai H, Ma Y, et al: Efficacy of posterior fossa decompression with duraplasty for patients with Chiari malformation type I: a systematic review and meta-analysis. World Neurosurg 113:357-365.e1, 2018

10. Chauvet D, Carpentier A, George B: Dura splitting decompression in Chiari type 1 malformation: clinical experience and radiological findings. Neurosurg Rev 32:465-470, 2009

11. Chotai S, Kshettry VR, Lamki T, Ammirati M: Surgical outcomes using wide suboccipital decompression for adult Chiari I malformation with and without syringomyelia. Clin Neurol Neurosurg 120:129-135, 2014

12. Durham SR, Fjeld-Olenec K: Comparison of posterior fossa decompression with and without duraplasty for the surgical treatment of Chiari malformation Type I in pediatric patients: a meta-analysis. J Neurosurg Pediatr 2:42-49, 2008

13. Eule JM, Erickson MA, O'Brien MF, Handler M: Chiari I malformation associated with syringomyelia and scoliosis: a twenty-year review of surgical and nonsurgical treatment in a pediatric population. Spine (Phila Pa 1976) 27:1451-1455, 2002

14. Genitori L, Peretta P, Nurisso C, Macinante L, Mussa F: Chiari type I anomalies in children and adolescents: minimally invasive management in a series of 53 cases. Childs Nerv Syst 16:707-718, 2000

15. Godzik J, Kelly MP, Radmanesh A, Kim D, Holekamp TF, Smyth MD, et al: Relationship of syrinx size and tonsillar descent to spinal deformity in Chiari malformation Type I with associated syringomyelia. J Neurosurg Pediatr 13:368-374, 2014 
16. Hankinson T, Tubbs RS, Wellons JC: Duraplasty or not? An evidence-based review of the pediatric Chiari I malformation. Childs Nerv Syst 27:35-40, 2011

17. Hankinson TC, Klimo P Jr, Feldstein NA, Anderson RC, Brockmeyer D: Chiari malformations, syringohydromyelia and scoliosis. Neurosurg Clin N Am 18:549-568, 2007

18. Heiss JD, Patronas N, DeVroom HL, Shawker T, Ennis R, Kammerer W, et al: Elucidating the pathophysiology of syringomyelia. J Neurosurg 91:553-562, 1999

19. Koueik J, Sandoval-Garcia C, Kestle JRW, Rocque BG, Frim DM, Grant GA, et al: Outcomes in children undergoing posterior fossa decompression and duraplasty with and without tonsillar reduction for Chiari malformation type I and syringomyelia: a pilot prospective multicenter cohort study. J Neurosurg Pediatr 25:21-29, 2019

20. Krieger MD, Falkinstein Y, Bowen IE, Tolo VT, McComb JG: Scoliosis and Chiari malformation Type I in children. J Neurosurg Pediatr 7:25-29, 2011

21. Ladner TR, Westrick AC, Wellons JC III, Shannon CN: Health-related quality of life in pediatric Chiari Type I malformation: the Chiari Health Index for Pediatrics. J Neurosurg Pediatr 17:76-85, 2016

22. Limonadi FM, Selden NR: Dura-splitting decompression of the craniocervical junction: reduced operative time, hospital stay, and cost with equivalent early outcome. J Neurosurg 101 (2 Suppl):184-188, 2004

23. Lin W, Duan G, Xie J, Shao J, Wang Z, Jiao B: Comparison of results between posterior fossa decompression with and without duraplasty for the surgical treatment of Chiari malformation type I: a systematic review and meta-analysis. World Neurosurg 110:460-474.e5, 2018

24. Litvack ZN, Lindsay RA, Selden NR: Dura splitting decompression for Chiari I malformation in pediatric patients: clinical outcomes, healthcare costs, and resource utilization. Neurosurgery 72:922-929, 2013

25. Lu VM, Phan K, Crowley SP, Daniels DJ: The addition of duraplasty to posterior fossa decompression in the surgical treatment of pediatric Chiari malformation Type I: a systematic review and meta-analysis of surgical and performance outcomes. J Neurosurg Pediatr 20:439-449, 2017

26. Sekula RF Jr, Arnone GD, Crocker C, Aziz KM, Alperin N: The pathogenesis of Chiari I malformation and syringomyelia. Neurol Res 33:232-239, 2011
27. Strahle J, Smith BW, Martinez M, Bapuraj JR, Muraszko KM, Garton HJ, et al: The association between Chiari malformation Type I, spinal syrinx, and scoliosis. J Neurosurg Pediatr 15:607-611, 2015

28. Williams H: A unifying hypothesis for hydrocephalus, Chiari malformation, syringomyelia, anencephaly and spina bifida. Cerebrospinal Fluid Res 5:7, 2008

29. Wu T, Zhu Z, Jiang J, Zheng X, Sun X, Qian B, et al: Syrinx resolution after posterior fossa decompression in patients with scoliosis secondary to Chiari malformation type I. Eur Spine J 21:1143-1150, 2012

30. Xu H, Chu L, He R, Ge C, Lei T: Posterior fossa decompression with and without duraplasty for the treatment of Chiari malformation type I-a systematic review and meta-analysis. Neurosurg Rev 40:213-221, 2017

31. Yilmaz A, Kanat A, Musluman AM, Colak I, Terzi Y, Kayac1 $S$, et al: When is duraplasty required in the surgical treatment of Chiari malformation type I based on tonsillar descending grading scale? World Neurosurg 75:307-313, 2011

\section{Disclosures}

Dr. Limbrick: support of non-study-related clinical or research effort from Medtronic and Microbot Medical.

\section{Author Contributions}

Conception and design: Hale, Park, Shannon, Limbrick. Acquisition of data: all authors. Analysis and interpretation of data: Hale, Limbrick. Drafting the article: Hale. Critically revising the article: all authors. Reviewed submitted version of manuscript: all authors. Approved the final version of the manuscript on behalf of all authors: Hale. Statistical analysis: Hale. Administrative/technical/material support: Shannon, Limbrick. Study supervision: Shannon, Limbrick.

\section{Correspondence}

Andrew T. Hale: Vanderbilt University School of Medicine, Nashville,TN. andrew.hale@vanderbilt.edu. 